\title{
Identifying and Correcting Step Losses in Single-Ended Fiber-Optic Distributed Temperature Sensing Data
}

\author{
Mark B. Hausner ${ }^{1}$ and Scott Kobs ${ }^{2}$ \\ ${ }^{1}$ Division of Hydrologic Sciences, Desert Research Institute, 755 East Flamingo Road, Las Vegas, NV 89119, USA \\ ${ }^{2}$ Department of Geological Sciences and Engineering, University of Nevada, Reno, MS 172, Reno, NV 89557, USA
}

Correspondence should be addressed to Mark B. Hausner; mark.hausner@dri.edu

Received 22 December 2015; Accepted 17 March 2016

Academic Editor: Kewei Zhang

Copyright (C) 2016 M. B. Hausner and S. Kobs. This is an open access article distributed under the Creative Commons Attribution License, which permits unrestricted use, distribution, and reproduction in any medium, provided the original work is properly cited.

Fiber-optic distributed temperature sensing (DTS) makes it possible to observe temperatures on spatial scales as fine as centimeters and at frequencies up to $1 \mathrm{~Hz}$. Over the past decade, fiber-optic DTS instruments have increasingly been employed to monitor environmental temperatures, from oceans to atmospheric monitoring. Because of the nature of environmental deployments, optical fibers deployed for research purposes often encounter step losses in the Raman spectra signal. Whether these phenomena occur due to cable damage or impingements, sharp bends in the deployed cable, or connections and splices, the step losses are usually not adequately addressed by the calibration routines provided by instrument manufacturers and can be overlooked in postprocessing calibration routines as well. Here we provide a method to identify and correct for the effects of step losses in raw Raman spectra data. The utility of the correction is demonstrated with case studies, including synthetic and laboratory data sets.

\section{Introduction}

Over the past decade, fiber-optic distributed temperature sensing (DTS) has increasingly been used in environmental monitoring applications. The technology, which was originally developed in the oil and gas industry [1], relies on Raman spectra scattering and time domain reflectometry to return spatially and temporally integrated temperatures along the length of a fiber-optic cable. With spatial sampling intervals as small as tens of centimeters and temporal resolution as fine as $1 \mathrm{~Hz}$, DTS has been a transformative technology in environmental temperature monitoring. Early environmental applications of DTS focused on in situ characterization of thermal [2] or hydraulic [3] formation properties, as well as detecting damaged power cables [4]. Since 2006, use of DTS for environmental monitoring has rapidly expanded, and recent applications include studies of groundwater flow and hydraulic properties [5-7], groundwater-surface water interactions [8-10], soil water content [11-13], atmospheric boundary layer phenomena [14-16], cryosphere studies [1719], and climate reconstructions [20]. Engineering applications of DTS include environmental fluid mechanics [21, 22], as well as detection of illicit storm sewer connections [23], combined sewer overflows [24], and leaking canals [25].

Many of the DTS instruments available on the market were originally designed to detect gross temperature changes that do not require fine temperature calibration: to detect damaged power lines, for example, an accuracy of $1^{\circ} \mathrm{C}$ was sufficient [4]. Environmental applications, however, often depend on the observation of small temperature differences or gradients in space and time. Although most commercially available instruments include onboard calibration routines, these methods seldom provide the required temperature precision [21, 26]. Through careful experimental design and postprocessing of the raw Raman spectra data, temperature differences as precise as tenths $[26,27]$ or even hundredths of a degree C [28] can be resolved.

DTS data rely on inelastic Raman spectra scattering to determine the temperatures of the optical fiber. The Raman spectra comprise two frequencies: the Raman Stokes and antiStokes signals, which are shifted by a predictable wavelength from the incident laser light [1]. The power of the backscattered Stokes signal, which has a slightly longer wavelength than incident, is largely temperature independent, while 


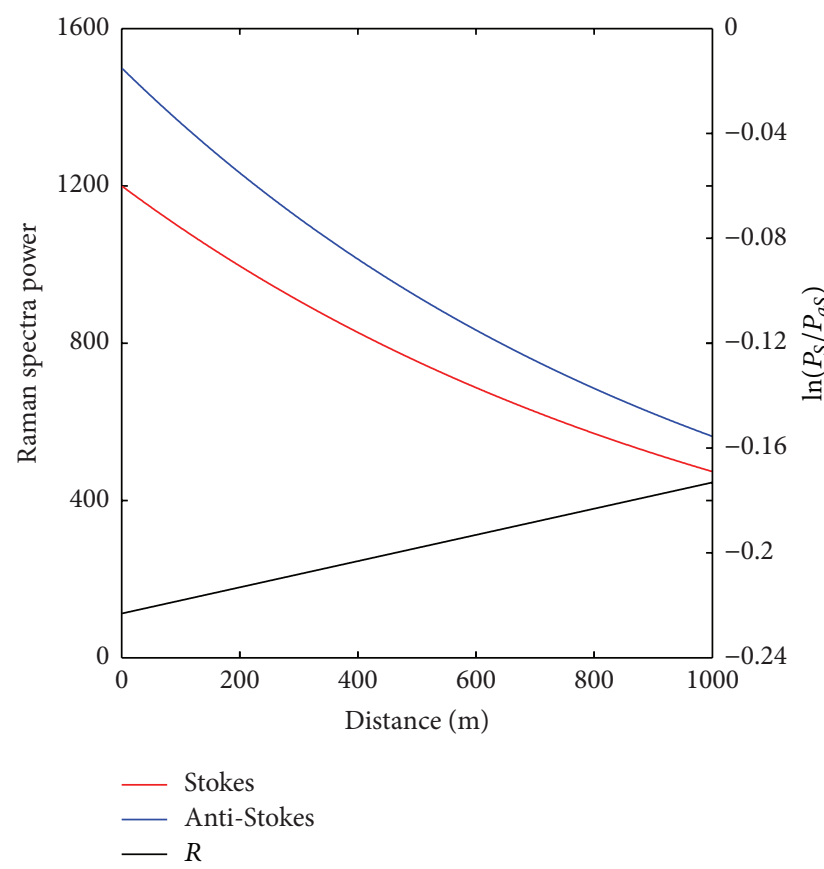

(a) Undamaged fiber at uniform temperature

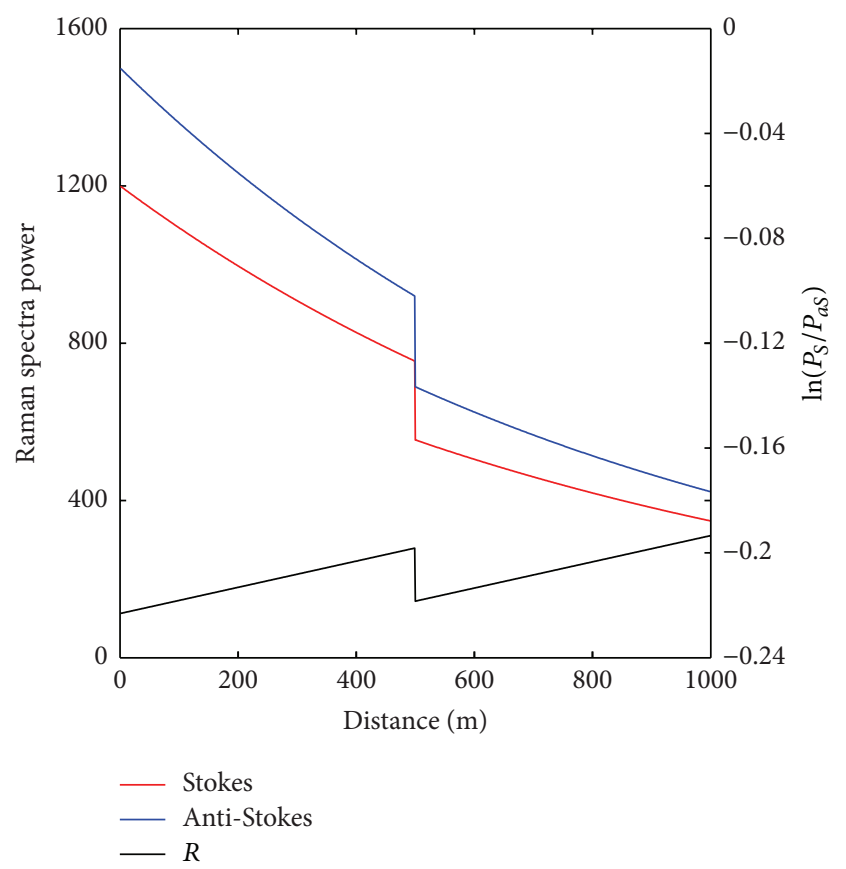

(b) Damaged fiber at uniform temperature

Figure 1: Sample Raman spectra data for a $1000 \mathrm{~m}$ fiber at uniform temperature. (a) An undamaged fiber with no step loss. (b) A fiber with a step loss at $500 \mathrm{~m}$.

the power of the anti-Stokes, which has a shorter wavelength than incident, is strongly dependent on the temperature of the fiber. Along with optical time domain reflectometry, the ratio of the Stokes and anti-Stokes signals can be used to return the integrated temperature of spatially distinct sections of the optical fiber [29]. With different wavelengths, the two Raman signals have distinct attenuation rates in an optical fiber, and calibration routines require knowledge of the differential attenuation rate $\Delta \alpha$ (the difference between the attenuation rates of the anti-Stokes and Stokes signals in a given glass fiber). Single-ended calibration routines assume that $\Delta \alpha$ is uniform over the entire length of the fiber [27], but $\Delta \alpha$ can exhibit local variations in field deployments.

The differential attenuation rate is a characteristic of each individual optical fiber and is affected by strains, impingements, sharp bends, and damage to the fiber itself [30]. When these phenomena occur at a point or over a short section on the fiber (the length of the sampling interval), step losses in the Raman Stokes and anti-Stokes signals occur (Figure 1). Step losses occur frequently in field applications of DTS, due to impingements on the fiber itself, sharp bends, consistent or transient stresses on the fiber, and damaged sections. In duplexed deployments, which comprise two fibers collocated in a single cable and connected at the far end, step losses can also be induced by the fusion splice or physical connectors (e.g., E2000 connectors) that join different lengths of optical fiber together. Tyler et al. [30] noted both the potential for these step losses to affect calibration and the importance of ensuring that they did not occur between the reference sections used to calibrate DTS data. In some cases, however, this occurrence is unavoidable.
If these step losses are not addressed in calibration, they can cause temperature errors that are small, sometimes even undetectable in the reference sections used to calibrate and validate the temperature data. On other parts of the cable, especially far from the calibration reference sections, the errors induced by neglecting step losses can be much greater. While double-ended calibration routines can account for step losses, those routines add noise to the data and are less precise than single-ended calibration methods, especially for shorter $(<2000 \mathrm{~m})$ cables [26,27]. A reliable method to identify and correct for step losses increases the applicability of singleended calibration and makes it possible to observe finer temperature changes and gradients within DTS data, even when losses in the backscatter data occur. Hausner et al. [27] corrected step losses by simply assuming that the ratio of the Raman signals should be the same on either side of the step loss, while Arnon et al. [28] employed an empirical correction to account for asymmetric losses in a tight-radius bend. Here we refine the method used by Hausner et al. [27] by including the influence of differential attenuation across the loss, and we present a method to identify step losses that may not be immediately apparent in the data. We provide the physical and theoretical justification for both the identification and correction methods and demonstrate the utility of the methods with synthetic and laboratory case studies.

\section{Methods}

DTS data represent temperatures integrated in both time and space [30]. For a defined integration time, temperatures on 
a section of fiber centered at distance $z$ from the instrument are determined from the raw Raman spectra data according to (1), in which $T$ is the temperature of the fiber; $P_{S}$ and $P_{a S}$ are the power of the Stokes and anti-Stokes signals, respectively; $z$ is the distance from the instrument; $\Delta \alpha$ is the differential attenuation; $C$ is a dimensionless calibration parameter describing the incident laser and photon detectors of the DTS instrument, and $\gamma(\mathrm{K})$ represents the change in energy between a photon at the incident wavelength and a scattered photon in the Raman spectra [27]. The calibration parameters $C$ and $\gamma$, which depend on characteristics of the instrument itself, are uniform over the entire fiber, but $\Delta \alpha$ frequently varies along the length of the cable:

$$
T(z)=\frac{\gamma}{\ln \left(P_{S}(z) / P_{a S}(z)\right)+C-\Delta \alpha z} .
$$

2.1. Identifying Step Losses. Many potential causes of step losses can be readily identified: splices, for example, occur at known points on a cable and the accompanying step losses can be easily identified in the raw data. Localized bending or strains may or may not be easily identified from a survey of the cable, and such phenomena may also be transient in nature. In these cases, a careful evaluation of the raw Raman spectra data may identify the location(s) of potential step losses. To identify potential step losses from the raw Raman trace, we examine the variance (standard deviation) of a tenpoint moving section of the cable.

Because the Raman anti-Stokes signal is more temperature sensitive than the Stokes signal, abrupt temperature changes (e.g., the cable entering or leaving a calibration bath) should induce a greater change in the anti-Stokes than the Stokes signal. In the case of step losses, however, the variance of the Stokes signal will be similar to that of anti-Stokes'. The potential step losses identified from this method may or may not be actual step losses, and further examination is required before any corrections are made. In some cases, this can be determined from the data themselves: if the anomaly occurs coincident with an increase in the anti-Stokes power, for example, it is due to warm spot on the fiber rather than a step loss. In other cases, it may not be apparent from the Raman spectra data and an examination of the cable itself is required. When this examination is not feasible and there is no other way to determine whether the anomaly is a step loss, researchers will have to use their best judgment in calibrating the data.

2.2. Correcting Step Losses. The simplest occurrence of a step loss is the case of a fiber at uniform temperature (i.e., $T(z)=$ $T_{0}$ ), such that the step loss results in a discontinuity of the function at that point. In this simple case, the step loss can be corrected by a uniform shift of the temperature data on the far side of the step loss. This simple correction can be applied to more complex data sets when two conditions are met:

(a) the temperature on either side of the step loss is equal (i.e., $T\left(z_{1}\right)=T\left(z_{2}\right)$ );

(b) the differential attenuation rate in the fiber is uniform except at the location of the step loss.
These two conditions allow the correction to be made to the transformed quantity $R(z)$ (see (2)), which is the natural log of the ratio of Stokes to anti-Stokes power. In the case of uniform temperature along an ideal, undamaged fiber, $R(z)$ is a linear function of distance (Figure 1(a)) [27]. A step loss in that uniform-temperature fiber that causes a discontinuity in the temperature function also results in a discontinuity in the function describing $R(z)$ : the slope of $R(z)$ remains constant except at the step loss, where an offset is seen (Figure 1(b)). At its most basic level, the correction method determines the magnitude of that offset and applies it to the entire length of fiber on the far side of the step loss:

$$
R(z)=\ln \left[\frac{P_{S}(z)}{P_{a S}(z)}\right] .
$$

Although step losses are typically caused by physical phenomena at a single point, the effects of the step losses are seen over multiple sampling intervals on either side of the point as well. To account for the spatial influence of the step loss, we locate the focal point of the step loss and move the same number of sampling intervals in either direction, to a sampling interval not influenced by the loss. This pair of points, an equal distance from the focus point, is used to determine the correction: we assume that the fiber at these two points is at the same temperature and rely on that assumption to calculate the magnitude of the offset shown in Figure 1(b). In a DTS trace with a $0.5 \mathrm{~m}$ sampling interval and a step loss focused at $500 \mathrm{~m}$, for example, we might use the observations located 5 sampling intervals before and after the step loss (i.e., $497.5 \mathrm{~m}$ and $502.5 \mathrm{~m}$ ) as the basis for the correction.

Assuming that these two points are at the same temperature, the expected difference in $R$ would be due solely to the differential attenuation (typically on the order of $10^{-5} \mathrm{~m}^{-1}$ ), and any greater difference is attributed to the step loss itself ((3), where $R\left(z_{1}\right)$ and $R\left(z_{2}\right)$ are the natural log of the ratios of the Raman signals at the chosen points before and after the step loss, resp., $\Delta \alpha$ is the differential attenuation rate, $\left(z_{2}-z_{1}\right)$ is the distance between the two chosen points, and $\Delta R_{\text {step }}$ represents the effect of the step loss). We estimate the differential attenuation rate based on the defined calibration sections for the cable: in those uniform-temperature sections, the slope of $R(z)$ is equal to the differential attenuation rate. The offset to account for the step loss then is determined by solving (3) for $\Delta R_{\text {step }}$ :

$$
R\left(z_{2}\right)=R\left(z_{1}\right)-\Delta \alpha\left(z_{2}-z_{1}\right)-\Delta R_{\text {step }}
$$

Once the correction offset $\Delta R_{\text {step }}$ is determined, that offset is applied to every observation on the far side of the step loss itself.

With the correction made to $R(z)$, the adjusted data are calibrated according to (1). Following the method of Hausner et al. [27], we use three sections of the cable at known temperatures to explicitly solve for the parameters $\gamma$, $C$, and $\Delta \alpha$. The precision of the calibration is measured using the root mean square error (RMSE) of the calibrated data within the reference sections. Accuracy of the calibration is 
TABLE 1: Synthetic data set parameters.

\begin{tabular}{lccr}
\hline Parameter & Units & Mean & Standard deviation \\
\hline$\gamma$ & $\mathrm{K}$ & 489.20 & 2.000 \\
$C$ & [Dimensionless] & 1.702 & 0.010 \\
$\Delta \alpha$ & $\mathrm{m}^{-1}$ & $5.001 \times 10^{-5}$ & $3.305 \times 10^{-5}$ \\
$a_{S}$ & $\mathrm{~m}^{-1}$ & $5.358 \times 10^{-4}$ & $2.032 \times 10^{-5}$ \\
$a_{a S}$ & $\mathrm{~m}^{-1}$ & $5.859 \times 10^{-4}$ & $2.688 \times 10^{-5}$ \\
$\ln \left(P_{S}\right)$ & {$[$ Dimensionless] } & $\mathrm{N} / \mathrm{A}$ & $4.09 \times 10^{-4}$ \\
$\ln \left(P_{a S}\right)$ & {$[$ Dimensionless] } & $\mathrm{N} / \mathrm{A}$ & $5.12 \times 10^{-4}$ \\
\hline
\end{tabular}

measured by the mean bias of validation reference sections: sections of the cable at known uniform temperatures that were not used in the calibration calculations.

\section{Data Sets}

The above methods are demonstrated using two data sets: a synthetic data set that demonstrates the need for and utility of the correction algorithm and a laboratory data set that demonstrates both the step loss detection procedure and the correction algorithm.

3.1. Synthetic Data Set. To demonstrate the correction, we created a synthetic data set based on the calibration parameters calculated for an existing DTS data set. The calibrated data set included 306 traces, and the calibrated values of $\gamma, C$, and $\Delta \alpha$, as well as the attenuation rates of the Stokes $\left(\alpha_{S}\right)$ and anti-Stokes $\left(\alpha_{a S}\right)$ signals, were examined. Each of those five parameters exhibited a normal distribution, and the means and standard deviations of those distributions were used to create the synthetic data set (Table 1$)$. In addition to those parameters, the standard deviations of $\ln \left(P_{S}\right)$ and $\ln \left(P_{a S}\right)$ were calculated for a uniform temperature. The data shown in Table 1 were used as the basis for the synthetic data set.

The synthetic data set included 1440 traces of an arbitrary, spatially variable temperature distribution that was assumed to be constant in time. For each trace, the values of $\gamma$, $C$, and $\Delta \alpha$ were determined randomly using the values in Table 1, with the restriction that value of $\Delta \alpha$ could not be less than zero. Based on these values and the known spatial temperature distribution $T(z)$, the corresponding spatially distributed values of $R(z)=\ln \left(P_{S}(z) / P_{a S}(z)\right)$ were determined using (1). The attenuation rate of either the Stokes or anti-Stokes signal was randomly determined, and the attenuation rate of the other was then calculated using the equation $\Delta \alpha=\alpha_{a S}-\alpha_{S}$ [21]. The value of the Stokes power $\left(P_{S}\right)$ at $z=0$ was arbitrarily set at 1000 , and the spatial distribution of $\ln \left[P_{S}(z)\right]$ was determined using the attenuation rate $\left(\alpha_{S}\right)$ and a temperature sensitivity of $0.01 \mathrm{~K}^{-1}$ ((4), where $T_{k}$ is the temperature in kelvins). The spatial distribution of $\ln \left[P_{a S}(z)\right]$ was then determined based on the temperature distribution, $\ln \left[P_{S}(z)\right], R(z)$, and (2). Normally distributed random errors were added to these values, and the exponential of each distribution was taken to return the synthetic distributions of Stokes and anti-Stokes power. The synthetic data set comprised the spatially variable, temporally constant temperature distribution, as well as the spatially and temporally variable distributions of Stokes and anti-Stokes power:

$$
\ln P_{S}(z)=\ln 1000-\alpha_{S} z+0.01\left[T_{k}(z)-298.15\right] .
$$

The complete synthetic data consisted of 1440 traces along a $1000 \mathrm{~m}$, duplexed cable recording a spatially variable temperature trace (Figure 2(a)) that remains constant in time. We compared the results of single-ended calibration routines applied to the ideal (no step loss) Raman spectra trace (Figure 2(b)) and both corrected and uncorrected routines applied to the trace with a step loss (Figure 2(c)). The synthetic temperature trace contained a number of uniformtemperature sections at a range of temperatures to make comparisons between different calibration routines easier. The synthetic data shown in Figure 2(c) were recalibrated using the three-section explicit method [27] both with and without the step loss correction. Each calibration was based on the reference sections from $1-24 \mathrm{~m}\left(25^{\circ} \mathrm{C}\right), 25-49 \mathrm{~m}\left(15^{\circ} \mathrm{C}\right)$, and $976-1000 \mathrm{~m}\left(25^{\circ} \mathrm{C}\right)$, and validation was done on the section $950-975 \mathrm{~m}\left(15^{\circ} \mathrm{C}\right)$. These reference sections were chosen to be representative of a typical duplexed calibration scheme.

Using the calibrated data, we calculated the RMSE and mean bias in the three calibration baths and the validation bath. We also calculate those parameters for the two passes through the mid-cable bath (475-499 $\mathrm{m}$ and 501-525 m).

3.2. Laboratory Testing. A laboratory experiment was conducted 6 March 2015 at the University of Nevada, Reno. A $125 \mathrm{~m}$ long, duplexed cable was deployed with three reference sections (Figures 5(a) and 5(b)), and the data were processed by the uncorrected and corrected calibration schemes using calibration reference sections $10-28 \mathrm{~m}$ $\left(\sim 28^{\circ} \mathrm{C}\right), 33-52 \mathrm{~m}\left(\sim 4^{\circ} \mathrm{C}\right)$, and $220-238 \mathrm{~m}\left(\sim 28^{\circ} \mathrm{C}\right)$ and validation section $197-213 \mathrm{~m}\left(\sim 4^{\circ} \mathrm{C}\right)$. DTS data were recorded with an Oryx (Sensornet Ltd., Hertfordshire, UK) using a $60 \mathrm{~s}$ integration time and a $1 \mathrm{~m}$ spatial sampling interval. Temperatures in the reference sections were measured independently with RBRsoloT temperature loggers (RBR Ltd., Kanata, Ontario, Canada) with NIST-traceable temperature accuracy of $\pm 0.002^{\circ} \mathrm{C}$. We calculated the RMSE and mean bias for the calibration and validation reference sections, as well as for two mid-cable sections (104-118 m and 129-144 m). 


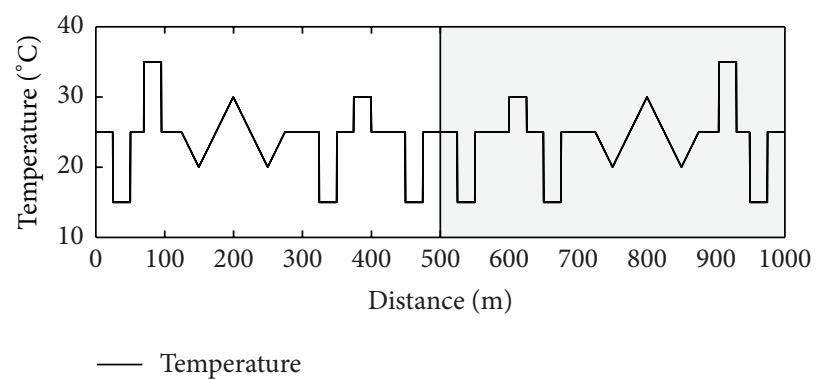

(a) Duplexed temperature trace

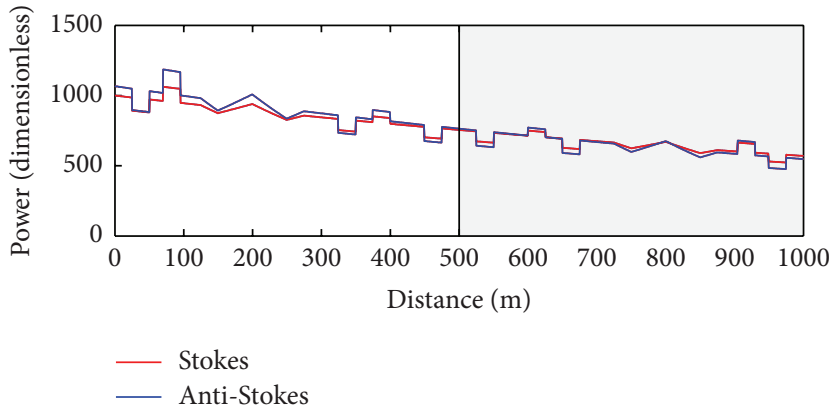

(b) Raman spectra raw data (no step losses)

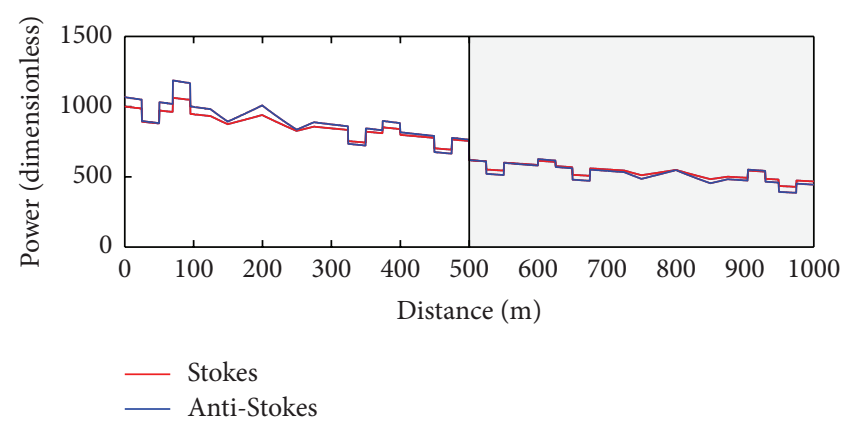

(c) Raman spectra raw data (typical step losses)

Figure 2: Synthetic data set. (a) Spatial distribution of temperatures used as the basis of the raw data. The data are duplexed about the midpoint of the fiber, as indicated by the shaded area on the right side of each plot. (b) An example of the ideal Raman spectra data for the specified temperature trace (no step loss). (c) A sample of the Raman spectra data including a step loss at $500 \mathrm{~m}$.

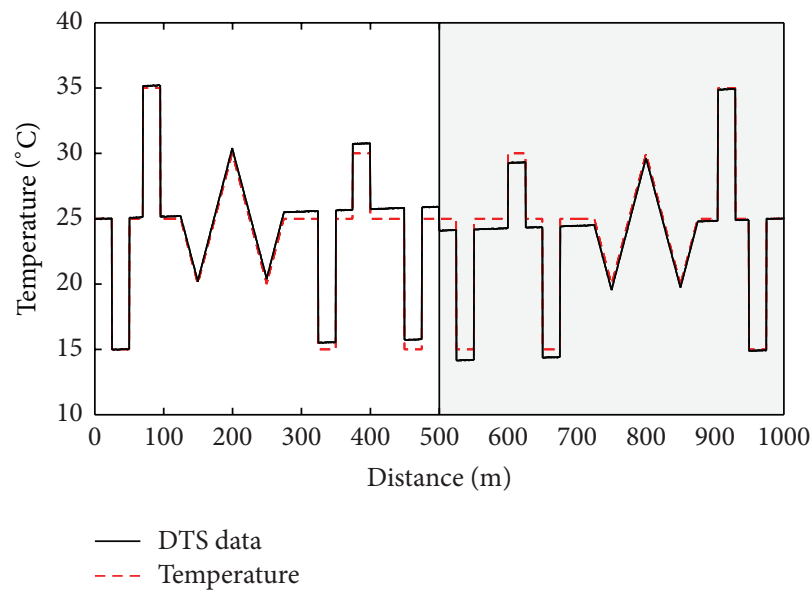

(a) Calibration neglecting step loss

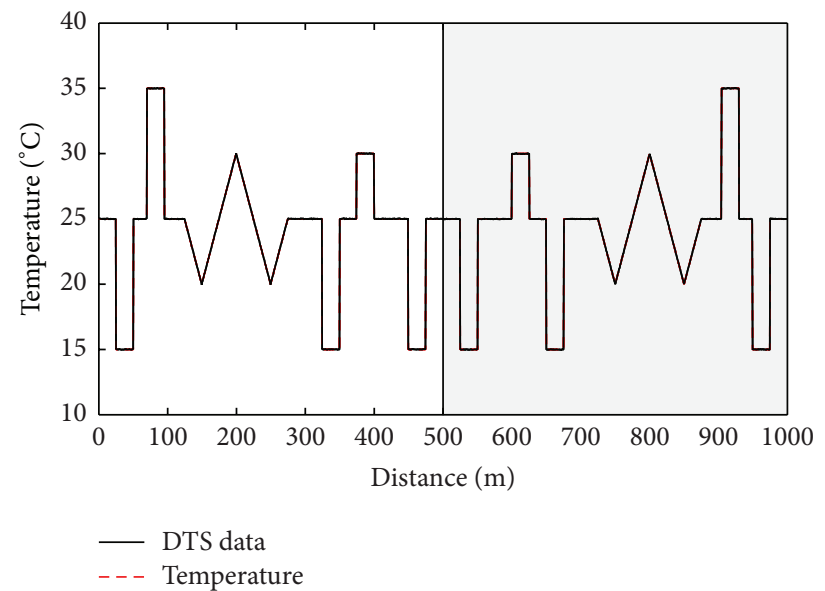

(b) Calibration correcting for step loss

FIGURE 3: Sample trace of recalibrated data for the synthetic data set including a step loss. (a) Uncorrected step loss. (b) Corrected step loss.

\section{Results}

Here we present the above methods applied to the different case studies. Because the locations of the step losses are known in the synthetic data set, we apply only the step loss correction. In the laboratory test, we apply both the identification algorithm and the correction algorithm.

4.1. Case Study 1: Synthetic Data Set. Calibration metrics were similar for both the corrected and uncorrected calibration schemes (Table 2). The difference between the two routines is apparent, though, in the validation metric. The uncorrected scheme has a mean bias of approximately the same magnitude as the validation RMSE, indicating that the uncorrected scheme consistently underestimates temperatures in the validation section. This discrepancy becomes much more apparent with the metrics from the mid-cable references: closer to the instrument $(475-499 \mathrm{~m})$ the temperatures are overestimated by almost $0.9^{\circ} \mathrm{C}$, while just $2 \mathrm{~m}$ away on the other side of the step loss $(501-525 \mathrm{~m})$ the temperature is underestimated by the same amount, resulting in an apparent $1.8^{\circ} \mathrm{C}$ discontinuity in the temperature trace. Calibration error 
TABLE 2: (a) Calibration, validation, and near-splice metrics from the uncorrected and corrected calibration schemes as applied to the synthetic data set. (b) Calibration, validation, and near-splice metrics from the corrected and uncorrected calibration schemes as applied to the laboratory test data.

(a)

\begin{tabular}{lccc}
\hline & & Uncorrected metrics $\mu \pm \sigma$ of 1440 traces ${ }^{\circ} \mathrm{C}$ & Corrected metrics $\mu \pm \sigma$ of 1440 traces ${ }^{\circ} \mathrm{C}$ \\
\hline \multirow{2}{*}{ Calibration } & RMSE & $0.023 \pm 0.001$ & $0.02 \pm 0.001$ \\
& Bias & $<10^{-5}$ & $<10^{-5}$ \\
\hline \multirow{2}{*}{ Validation } & RMSE & $0.09 \pm 0.005$ & $0.02 \pm 0.002$ \\
& Bias & $-0.09 \pm 0.005$ & $0 \pm 0.005$ \\
\hline \multirow{2}{*}{$475-499 \mathrm{~m}$} & RMSE & $0.89 \pm 0.005$ & $0.02 \pm 0.005$ \\
& Bias & $0.89 \pm 0.005$ & $0.00 \pm 0.01$ \\
\hline \multirow{2}{*}{$501-525 \mathrm{~m}$} & RMSE & $0.89 \pm 0.005$ & $0.02 \pm 0.005$ \\
& Bias & $-0.89 \pm 0.005$ & $0.00 \pm 0.01$ \\
\hline
\end{tabular}

(b)

\begin{tabular}{lccc}
\hline & & Uncorrected metrics $\mu \pm \sigma$ of 208 traces ${ }^{\circ} \mathrm{C}$ & Corrected metrics $\mu \pm \sigma$ of 208 traces ${ }^{\circ} \mathrm{C}$ \\
\hline \multirow{2}{*}{ Calibration } & RMSE & $0.10 \pm 0.02$ & $0.09 \pm 0.02$ \\
& Bias & $<10^{-4}$ & $<10^{-4}$ \\
\hline \multirow{2}{*}{ Validation } & RMSE & $0.20 \pm 0.03$ & $0.15 \pm 0.05$ \\
& Bias & $0.14 \pm 0.02$ & $0.03 \pm 0.03$ \\
\hline \multirow{2}{*}{$104-118 \mathrm{~m}$} & RMSE & $0.38 \pm 0.02$ & $0.14 \pm 0.04$ \\
& Bias & $-0.39 \pm 0.02$ & $-0.13 \pm 0.04$ \\
\hline \multirow{2}{*}{$129-144 \mathrm{~m}$} & RMSE & $0.14 \pm 0.02$ & $0.15 \pm 0.05$ \\
& Bias & $0.12 \pm 0.02$ & $-0.13 \pm 0.06$ \\
\hline
\end{tabular}

increases with distance from the DTS instrument, reaching a maximum at the location of the step loss (Figure 3(a)).

In the corrected scheme, the RMSE and mean bias of the validation bath are the same as those in the calibration baths. The mid-cable baths, too, have RMSE values identical to the RMSE of the calibration baths and show no bias. Figure 3 shows the differences between the uncorrected calibration and the corrected calibration (Figure 3(b)), which has a consistent RMSE and zero bias over the entire length of the cable.

4.2. Case Study 2: Laboratory Data. Figure 4 shows the actual temperature (Figure 4(a)), mean Stokes and antiStokes Raman signals (Figure 4(b)), and the variance of the ten-point moving section used to identify step losses. The running variance of the Raman signals increases at a number of points along the fiber. When increases are caused by an abrupt temperature change, the variance of the anti-Stokes signal will increase more than the variance of the Stokes signal. This response is seen at $30 \mathrm{~m}, 54 \mathrm{~m}, 99 \mathrm{~m}, 147 \mathrm{~m}, 192 \mathrm{~m}$, and $216 \mathrm{~m}$ (Figure 4(c)). Those locations all correspond to abrupt temperature changes where the cable enters or leaves a calibration bath (Figure 4(a)), and the temperature change is also apparent in the raw Raman signals (Figure 4(b)). At several points on the trace shown in Figure 2(c), however, the variance of the Stokes signal is the same as or greater than the variance of the anti-Stokes signal. These spikes occur at $5 \mathrm{~m}, 123 \mathrm{~m}$, and $244 \mathrm{~m}$ (Figure 2(c)) and correspond to the connection between the first fiber and the DTS instrument, the fusion splice at the end of the duplexed cable, and the connection between the instrument and the second fiber, respectively. Each of these points induces a step loss, from either the physical connection or the fusion splice, and can be identified by this method.

Table 2(b) shows the calibration and validation metrics for the laboratory data. With the uncorrected calibration routine, the validation bath consistently overestimates the temperature (mean bias $0.14 \pm 0.02^{\circ} \mathrm{C}$, compared to the calibration RMSE of $0.10 \pm 0.02^{\circ} \mathrm{C}$ ), and the mid-cable reference bath shows the same pattern seen in the synthetic data. In this case, the near-instrument section (104-118 m) underestimates the temperature by $0.4^{\circ} \mathrm{C}$, while temperature in the same bath on the other side of the splice (129-144 m) is overestimated by $0.12^{\circ} \mathrm{C}$.

As in the synthetic data set, correcting for the step loss in the splice improves the calibrated data. In this case, the validation RMSE falls to $0.15^{\circ} \mathrm{C}$, and the magnitude of the mean bias in both of the mid-cable baths is less than this RMSE. The correction also ensures that the bias in the baths on either side of the step loss is identical.

\section{Discussion}

The loss correction algorithm presented here was designed for and works best with duplexed installations such as those recommended by NSF CTEMPs and by Hausner et al. [27]. Duplexed deployments allow the collection of both singleended and double-ended data. However, duplexed cables typically include a fusion splice at one end of the cable, which induces a step loss in the Raman spectra data. Because 


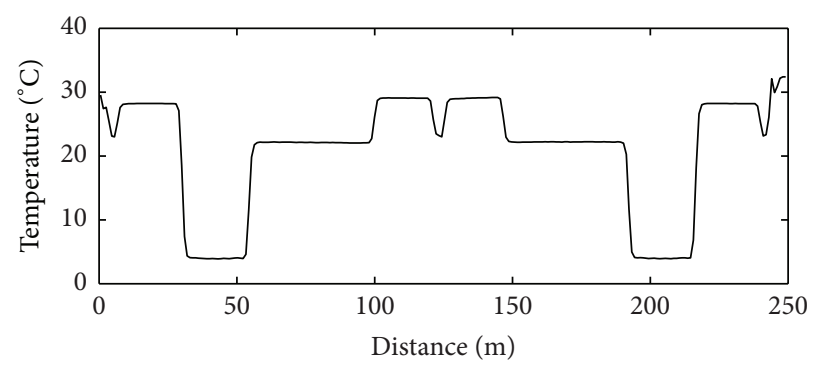

(a) Corrected temperature trace

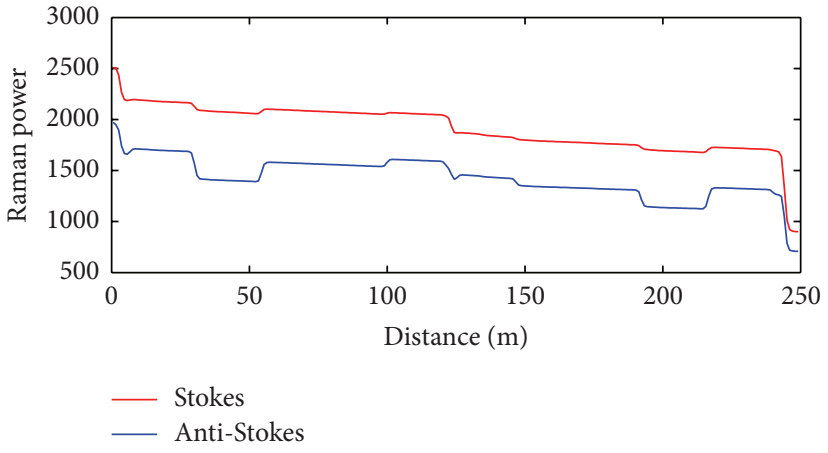

(b) Raman spectra signals

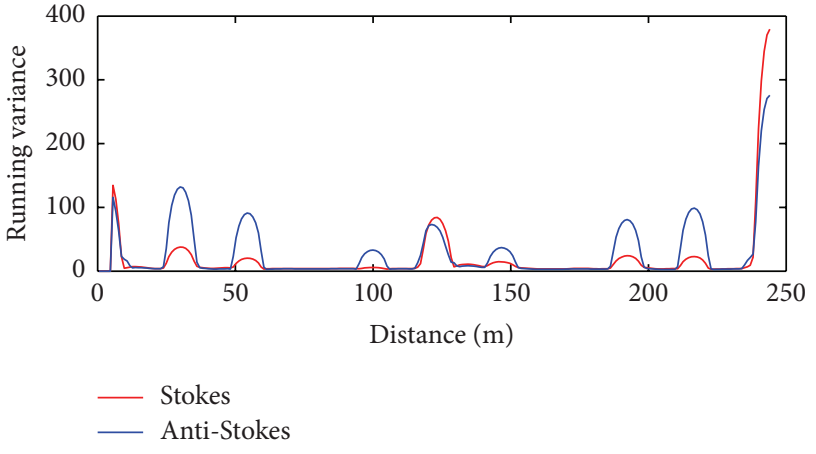

(c) Raman spectra running variance

FIgURE 4: Identifying step losses from the Raman spectra signals. (a) Corrected temperature trace along the length of the cable. (b) Raw Raman spectra Stokes and anti-Stokes data. (c) 10-point running variance of the raw Raman spectra data.

the nature of the duplexing makes the assumption that the temperature is the same on both sides of the step loss valid, it is prudent to compare single-ended and double-ended calibration routines and choose the method that returns more reliable data.

The synthetic and laboratory data presented here ranged between approximately $4^{\circ} \mathrm{C}$ and $30^{\circ} \mathrm{C}$, and the reference sections in each case study represent the minimum and maximum temperatures encountered. This is important to the calibration routine: calibrated data are most reliable within the temperature range between the warmest and coolest reference sections. Experimental designs should consider this and select reference sections accordingly.

In cases where a cable is damaged and must be repaired in the field, the repair will induce a step loss as well. If possible, a length of cable should be included on either side of the repaired fiber to ensure that this correction method can be used. In other cases, step losses can be induced by transient phenomena (bends tensioned by wind, e.g., or cables stressed by fallen trees). In these cases, this correction routine may or may not be applicable, depending on the uniformity of temperature around the stressed location and the precision of temperature observations required by the application. For step losses that can be predicted and accounted for in the experimental design, however, this correction method can greatly improve the quality of the data.

\section{Conclusions}

The effects of step losses, especially step losses far from the DTS instrument, can be neglected by conventional calibration routines and are not always readily identified unless they are specifically considered. The methodology presented here can be used to identify likely locations of step losses on an inaccessible cable, but each plausible step loss should be carefully considered by researchers before any correction is applied: the identification is a first step only. If the identified areas are determined to be step losses, the effects of those step losses can be corrected during calibration.

When the correction algorithm is applied, temperatures near the point of the step loss, in particular, are improved. In many applications (e.g., downhole experiments), these temperatures are critical to the study, and improved confidence in these regions can lead to better data and less uncertainty in experimental results. Careful experimental design and evaluation of all calibration options can greatly improve the reliability and utility of DTS data for environmental monitoring. We recommend that researchers be aware of and consider the following issues when planning a DTS deployment:

(i) selecting an appropriate fiber-optic cable that provides suitable protection and strain relief for the optical fiber,

(ii) careful cable handling during deployment and repair, with independent strain relief and anchors where necessary,

(iii) duplexed deployments to ensure that both singleended and double-ended calibrations can be applied to the collected data, 


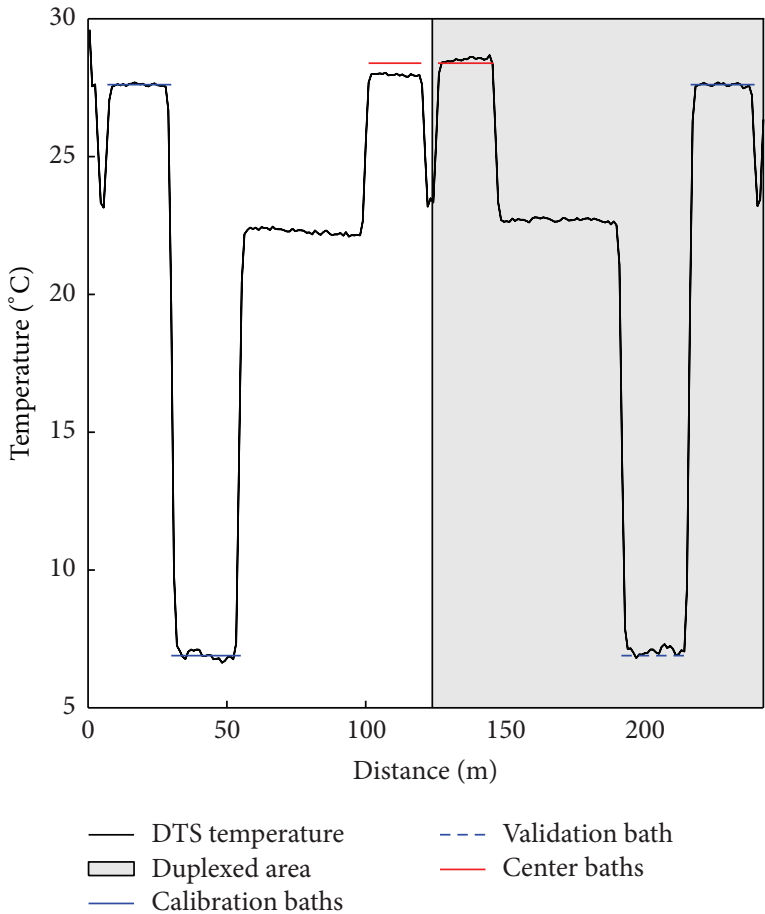

(a) Uncorrected calibration

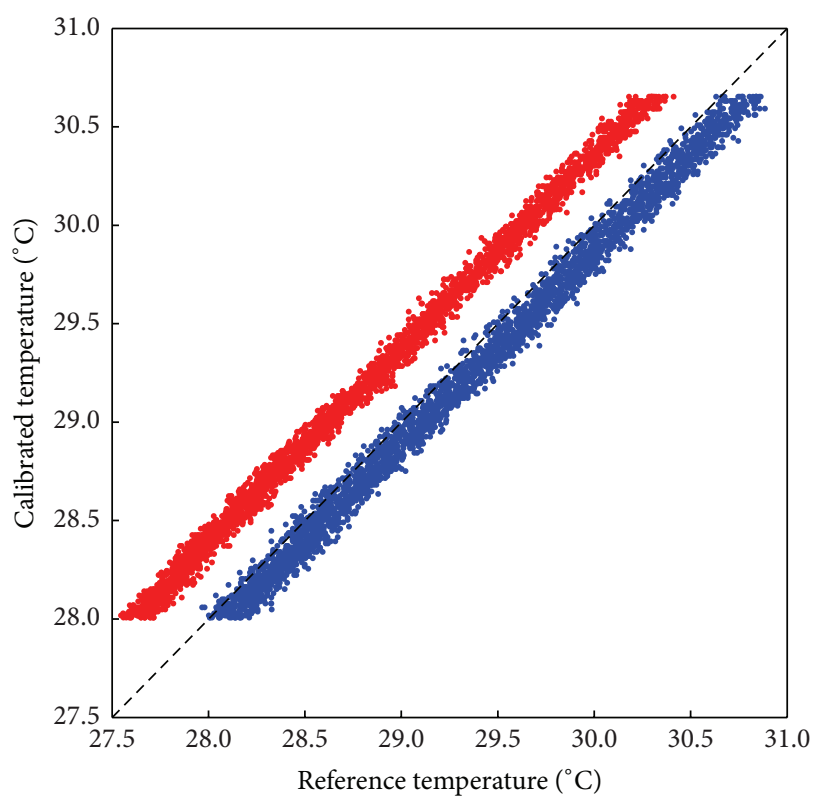

- $104-118 \mathrm{~m}$

- $129-144 \mathrm{~m}$

(c) Uncorrected calibration

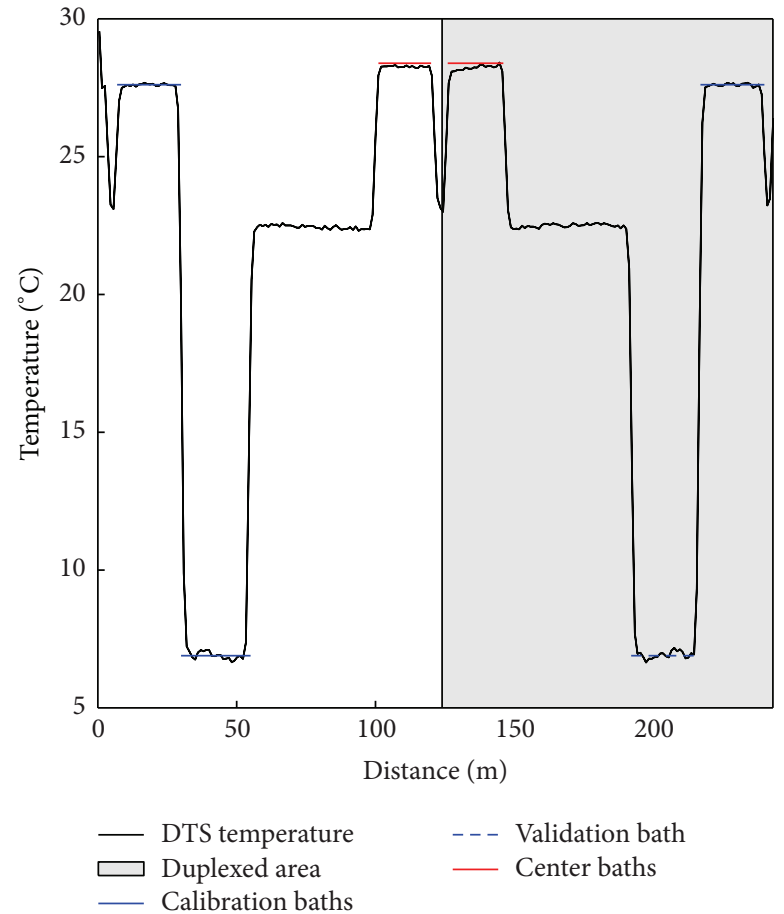

(b) Corrected calibration

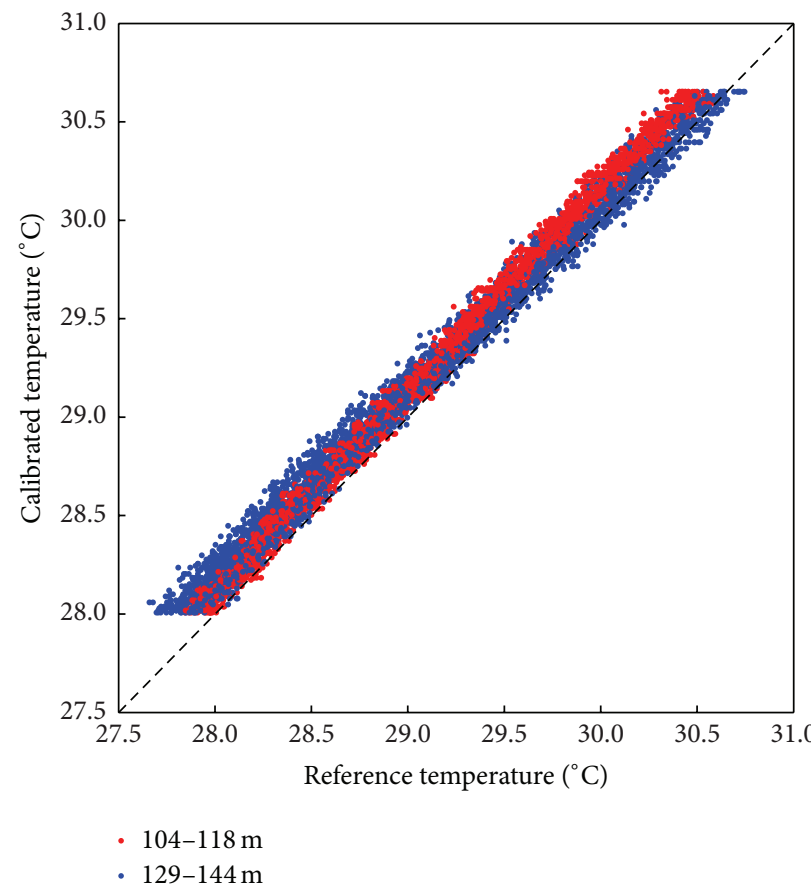

(d) Corrected calibration

Figure 5: Calibrated DTS temperatures with different calibration schemes applied to the field data set. (a) Temperature along the entire cable, uncorrected calibration. (b) Temperature along the entire fiber, corrected calibration. (c) Scatter plot of reference and calibrated temperatures in the middle of the cable, uncorrected calibration. (d) Scatter plot of reference and calibrated temperatures in the middle of the cable, corrected calibration. 
(iv) placement of calibration and validation reference sections at locations both near and far from the DTS instrument,

(v) designing reference sections such that the reference temperatures bracket the temperatures expected during observations,

(vi) careful planning and completion of any required repairs, including reference section at the same temperature on either side of any repair splices.

These recommendations make both single-ended and doubleended calibrations possible. While double-ended calibrations can usually handle localized step losses accurately [26], those routines are less precise than single-ended methods $[26,27,30]$, especially for short cables [27]. The step loss correction presented here will allow researchers the option to use single-ended calibration methods to refine DTS data from deployments with localized losses.

\section{Competing Interests}

The authors state that there are no competing interests related to this paper.

\section{Acknowledgments}

This work was made possible by CTEMPs (NSF EAR1128999 ) and by the generosity and support of the Sulo and Aileen Maki Endowment.

\section{References}

[1] J. S. Selker, L. Thévenaz, H. Huwald et al., "Distributed fiber-optic temperature sensing for hydrologic systems," Water Resources Research, vol. 42, no. 12, Article ID W12202, 2006.

[2] U. Günzel and H. Wilhelm, "Estimation of the in-situ thermal resistance of a borehole using the distributed temperature sensing (DTS) technique and the temperature recovery method (TRM)," Geothermics, vol. 29, no. 6, pp. 689-700, 2000.

[3] E. Hurtig, S. Grosswig, and K. Kühn, "Distributed fibre optic temperature sensing: a new tool for long-term and short-term temperature monitoring in boreholes," Energy Sources, vol. 19, no. 1, pp. 55-62, 1997.

[4] G. Yilmaz and S. E. Karlik, "A distributed optical fiber sensor for temperature detection in power cables," Sensors and Actuators A, vol. 125, no. 2, pp. 148-155, 2006.

[5] A. T. Leaf, D. J. Hart, and J. M. Bahr, "Active thermal tracer tests for improved hydrostratigraphic characterization," Groundwater, vol. 50, no. 5, pp. 726-735, 2012.

[6] T. Read, O. Bour, V. Bense et al., "Characterizing groundwater flow and heat transport in fractured rock using fiber-optic distributed temperature sensing," Geophysical Research Letters, vol. 40, no. 10, pp. 2055-2059, 2013.

[7] E. W. Banks, M. A. Shanafield, and P. G. Cook, "Induced temperature gradients to examine groundwater flowpaths in open boreholes," Groundwater, vol. 52, no. 6, pp. 943-951, 2014.

[8] M. C. Westhoff, H. H. G. Savenije, W. M. J. Luxemburg et al., "A distributed stream temperature model using high resolution temperature observations," Hydrology and Earth System Sciences, vol. 11, pp. 1469-1480, 2007.
[9] K. B. Moffett, S. W. Tyler, T. Torgersen, M. Menon, J. S. Selker, and S. M. Gorelick, "Processes controlling the thermal regime of saltmarsh channel beds," Environmental Science and Technology, vol. 42, no. 3, pp. 671-676, 2008.

[10] R. D. Henderson, F. D. Day-Lewis, and C. F. Harvey, "Investigation of aquifer-estuary interaction using wavelet analysis of fiber-optic temperature data," Geophysical Research Letters, vol. 36, no. 6, Article ID L06403, 2009.

[11] C. Sayde, C. Gregory, M. Gil-Rodriguez et al., "Feasibility of soil moisture monitoring with heated fiber optics," Water Resources Research, vol. 46, no. 6, Article ID W06201, 2010.

[12] S. C. Steele-Dunne, M. M. Rutten, D. M. Krzeminska et al., "Feasibility of soil moisture estimation using passive distributed temperature sensing," Water Resources Research, vol. 46, no. 3, Article ID W03534, 2010.

[13] A. M. Striegl and S. P. Loheide II, "Heated distributed temperature sensing for field scale soil moisture monitoring," Ground Water, vol. 50, no. 3, pp. 340-347, 2012.

[14] M. J. Zeeman, J. S. Selker, and C. K. Thomas, "Near-surface motion in the nocturnal, stable boundary layer observed with fibre-optic distributed temperature sensing," Boundary-Layer Meteorology, vol. 154, no. 2, pp. 189-205, 2014.

[15] C. A. Keller, H. Huwald, M. K. Vollmer et al., "Fiber optic distributed temperature sensing for the determination of the nocturnal atmospheric boundary layer height," Atmospheric Measurement Techniques, vol. 4, no. 2, pp. 143-149, 2011.

[16] C. K. Thomas, A. M. Kennedy, J. S. Selker et al., "Highresolution fibre-optic temperature sensing: a new tool to study the two-dimensional structure of atmospheric surface-layer flow," Boundary-Layer Meteorology, vol. 142, no. 2, pp. 177-192, 2012.

[17] S. W. Tyler, S. A. Burak, J. P. McNamara, A. Lamontagne, J. S. Selker, and J. Dozier, "Spatially distributed temperatures at the base of two mountain snowpacks measured with fiber-optic sensors," Journal of Glaciology, vol. 54, no. 187, pp. 673-679, 2008.

[18] S. W. Tyler, D. M. Holland, V. Zagorodnov et al., "Instruments and methods using distributed temperature sensors to monitor an Antarctic ice shelf and sub-ice-shelf cavity," Journal of Glaciology, vol. 59, no. 215, pp. 583-591, 2013.

[19] S. Kobs, D. M. Holland, V. Zagorodnov, A. Stern, and S. W. Tyler, "Novel monitoring of Antarctic ice shelf basal melting using a fiber-optic distributed temperature sensing mooring," Geophysical Research Letters, vol. 41, no. 19, pp. 6779-6786, 2014.

[20] B. M. Freifeld, S. Finsterle, T. C. Onstott, P. Toole, and L. M. Pratt, "Ground surface temperature reconstructions: using in situ estimates for thermal conductivity acquired with a fiberoptic distributed thermal perturbation sensor," Geophysical Research Letters, vol. 35, no. 14, Article ID L14309, 2008.

[21] F. Suárez, J. E. Aravena, M. B. Hausner, A. E. Childress, and S. W. Tyler, "Assessment of a vertical high-resolution distributedtemperature-sensing system in a shallow thermohaline environment," Hydrology and Earth System Sciences, vol. 15, no. 3, pp. 1081-1093, 2011.

[22] M. B. Hausner, K. P. Wilson, D. B. Gaines, and S. W. Tyler, "Interpreting seasonal convective mixing in Devils Hole, Death Valley National Park, from temperature profiles observed by fiber-optic distributed temperature sensing," Water Resources Research, vol. 48, no. 5, Article ID W05513, 2012.

[23] O. A. C. Hoes, R. P. S. Schilperoort, W. M. J. Luxemburg, F. H. L. R. Clemens, and N. C. van de Giesen, "Locating illicit 
connections in storm water sewers using fiber-optic distributed temperature sensing," Water Research, vol. 43, no. 20, pp. 51875197, 2009.

[24] R. P. S. Schilperoort and F. H. L. R. Clemens, "Fibre-optic distributed temperature sensing in combined sewer systems," Water Science and Technology, vol. 60, no. 5, pp. 1127-1134, 2009.

[25] O. A. C. Hoes, W. M. J. Luxemburg, M. C. Westhof, N. C. Van De Giesen, and J. Selker, "Identifying seepage in ditches and canals in polders in the netherlands by distributed temperature sensing," Lowland Technology International, vol. 11, no. 2, pp. 2126, 2009.

[26] N. van de Giesen, S. C. Steele-Dunne, J. Jansen et al., "Doubleended calibration of fiber-optic raman spectra distributed temperature sensing data," Sensors, vol. 12, no. 5, pp. 5471-5485, 2012.

[27] M. B. Hausner, F. Suárez, K. E. Glander, N. van de Giesen, J. S. Selker, and S. W. Tyler, "Calibrating single-ended fiber-optic Raman spectra distributed temperature sensing data," Sensors, vol. 11, no. 11, pp. 10859-10879, 2011.

[28] A. Arnon, J. Selker, and N. Lensky, "Correcting artifacts in transition to a wound optic fiber: example from high-resolution temperature profiling in the Dead Sea," Water Resources Research, vol. 50, no. 6, pp. 5329-5333, 2014.

[29] M. A. Farahani and T. Gogolla, "Spontaneous Raman scattering in optical fibers with modulated probe light for distributed temperature Raman remote sensing," Journal of Lightwave Technology, vol. 17, no. 8, pp. 1379-1391, 1999.

[30] S. W. Tyler, J. S. Selker, M. B. Hausner et al., "Environmental temperature sensing using Raman spectra DTS fiber-optic methods," Water Resources Research, vol. 46, no. 4, Article ID W00D23, 2010. 


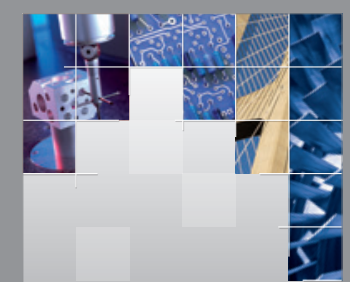

\section{Enfincering}
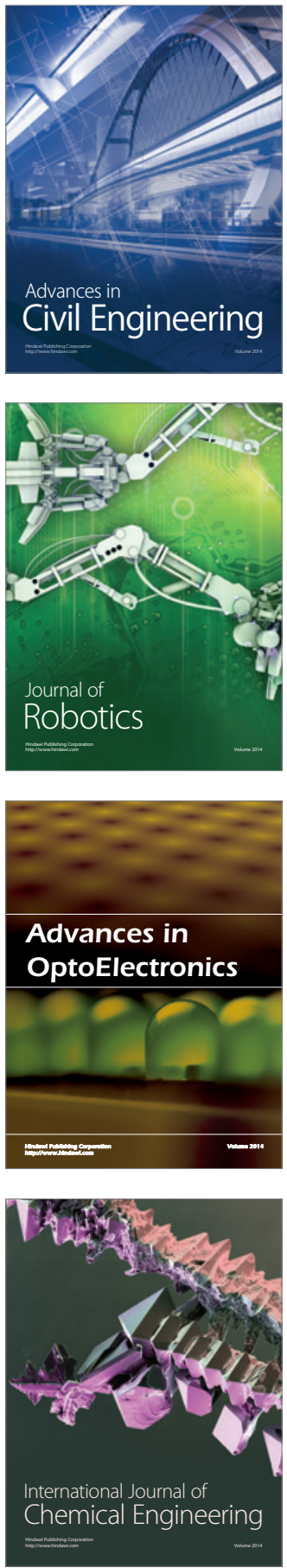

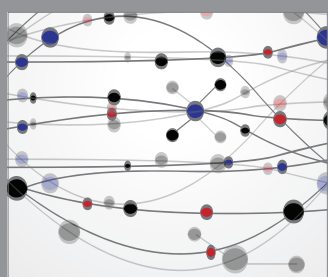

The Scientific World Journal

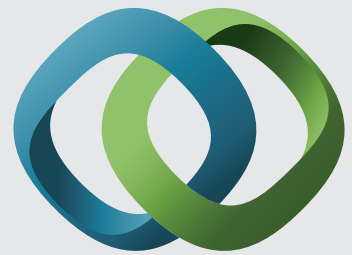

\section{Hindawi}

Submit your manuscripts at

http://www.hindawi.com
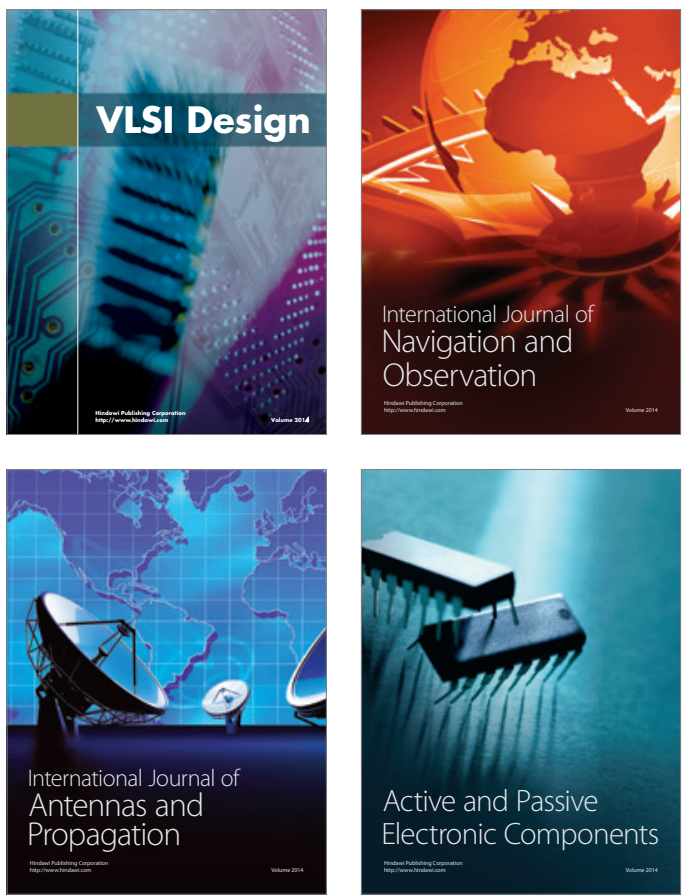
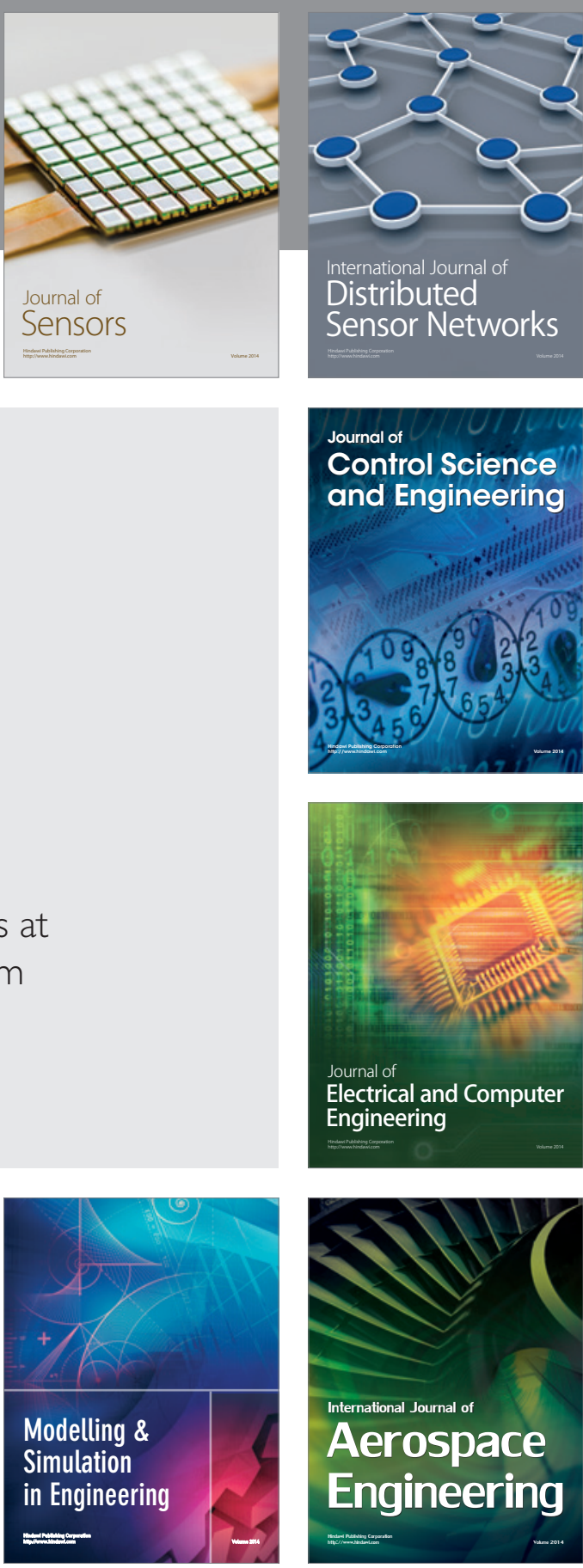

International Journal of

Distributed

Sensor Networks

Journal of

Control Science

and Engineering
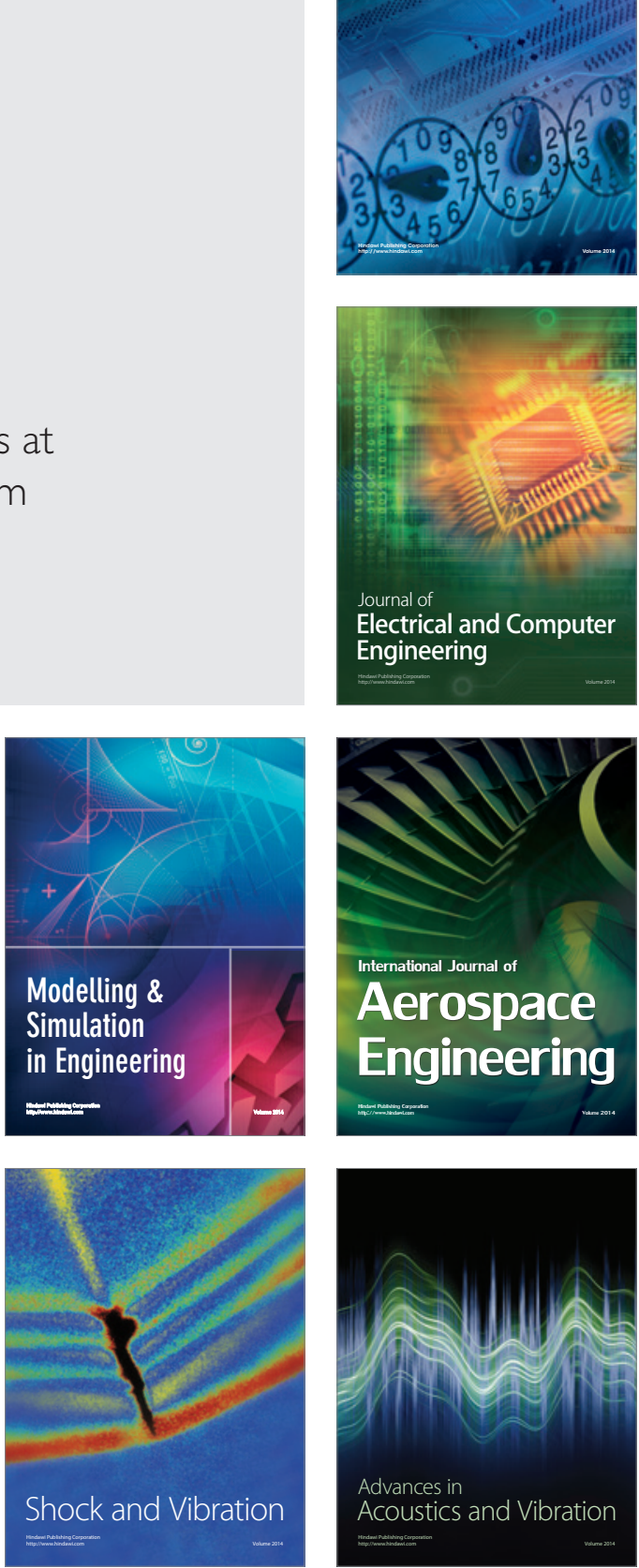